OPEN ACCESS

Edited by:

Marloes Dekker Nitert,

The University of

Queensland, Australia

Reviewed by:

Hongting Zheng,

Xinqiao Hospital, China

Bert Groen,

Academic Medical Research

(AMR), Netherlands

Houkai Li,

Shanghai University of Traditional

Chinese Medicine, China

*Correspondence:

Jia-Xing Tian

tina_yai@126.com

Xiao-lin Tong

tongxiaolin@vip.163.com

Specialty section:

This article was submitted to Microbiome in Health and Disease,

a section of the journal

Frontiers in Cellular and Infection

Microbiology

Received: 12 March 2020 Accepted: 16 September 2020

Published: 23 October 2020

Citation:

Ding Q-Y, Tian J-X, Li M, Lian F-M,

Zhao L-H, Wei X-X, Han L, Zheng Y-J,

Gao Z-Z, Yang $H-Y$, Fang $X-Y$ and

Tong $X-I$ (2020) Interactions Between

Therapeutics for Metabolic Disease,

Cardiovascular Risk Factors, and

Gut Microbiota.

Front. Cell. Infect. Microbiol.

10:530160

doi: 10.3389/fcimb.2020.530160

\section{Interactions Between Therapeutics for Metabolic Disease, Cardiovascular Risk Factors, and Gut Microbiota}

\author{
Qi-You Ding ${ }^{1,2}$, Jia-Xing Tian ${ }^{1 *}$, Min Li ${ }^{1}$, Feng-Mei Lian ${ }^{1}$, Lin-Hua Zhao ${ }^{1}$, Xiu-Xiu Wei ${ }^{1,2}$, \\ Lin Han ${ }^{1}$, Yu-Jiao Zheng ${ }^{1,2}$, Ze-Zheng Gao ${ }^{1,2}$, Hao-Yu Yang ${ }^{1,2}$, Xin-Yi Fang ${ }^{1,2}$ and \\ Xiao-lin Tong ${ }^{1 *}$ \\ 1 Department of Endocrinology, Guang'anmen Hospital, China Academy of Chinese Medical Sciences, Beijing, China, \\ ${ }^{2}$ Graduate College, Beijing University of Traditional Chinese Medicine, Beijing, China
}

With improved standards of living, the incidence of multiple metabolic disorders has increased year by year, especially major risk factors for cardiovascular disease such as hyperglycemia and hyperlipidemia, continues to increase. Emerging epidemiological data and clinical trials have shown the additional protective effects of some metabolic therapy drugs against cardiovascular diseases. A series of studies have found that these drugs may work by modulating the composition of gut microbiota. In this review, we provide a brief overview of the contribution of the gut microbiota to both metabolic disorders and cardiovascular diseases, as well as the response of gut microbiota to metabolic therapy drugs with cardiovascular benefits. In this manner, we link the recent advances in microbiome studies on metabolic treatment drugs with their cardiovascular protective effects, suggesting that intestinal microorganisms may play a potential role in reducing cardiovascular risk factors. We also discuss the potential of microorganism-targeted therapeutics as treatment strategies for preventing and/or treating cardiovascular disease and highlight the need to establish causal links between therapeutics for metabolic diseases, gut microbiota modulation, and cardiovascular protection.

Keywords: metabolic therapy, gut microbiota, cardiovascular diseases, metformin, microbiota-targeted therapies

\section{INTRODUCTION}

Human dietary habits and lifestyle have changed greatly over time with the development of society (Chauveau et al., 2013). At the same time, the number of people suffering from metabolic diseases such as type 2 diabetes mellitus (T2DM), obesity, and dyslipidemia has also grown rapidly (Whiting et al., 2011; Loh et al., 2019). These metabolic disorders have proven to be major risk factors for cardiovascular diseases (CVD) (DeFronzo and Ferrannini, 1991; Barr et al., 2007). Despite adherence to recommended therapies, especially multi-drug combination therapy for patients with multiple metabolic disorders, treatment response is individual and varied. Many individuals remain at a high risk of developing CVD. Although genetic factors play a role in CVD pathogenesis, large-scale studies have revealed that they may only account 
for $20 \%$ of the risk of developing CVD (Ripatti et al., 2010). Thus, environmental factors play a dominant role in CVD pathogenesis.

Gut microbiota are an important environmental factor, and there is increasing evidence that the activity and composition of the gut microbiota are closely related to human diseases (Clemente et al., 2012; Sonnenburg and Backhed, 2016). It is believed that gut microbiota participate in the absorption of nutrients and energy, engage the innate immune system, and produce a wide variety of small-molecule metabolites that are sensed by host receptor systems to regulate host metabolism and inflammatory pathways relevant to CVD (Tang et al., 2017; Brown and Hazen, 2018). In the treatment of CVD, lowering risk factors such as hyperglycemia and hyperlipidemia, has been shown to reduce cardiovascular events (Grundy et al., 2004; Mazzone et al., 2008; Mooradian, 2009; Eliasson et al., 2011). In parallel, many metabolic disease treatments have also been shown to work through the gut microbiota. With the discovery of more microbial targets, an improved understanding of the specific effects of metabolic therapy drugs on gut microbiota is still required. This may also provide a new treatment strategy for the control of CVD risk factors.

In this review, we provide a brief overview on the contribution of the gut microbiota to metabolic disorders as well as CVD, and the response of gut microbiota to cardiovascular metabolic therapeutic drugs. In this manner, we link the existing studies on gut microbiota regulation by metabolic therapy drugs with their cardiovascular protective effects, suggesting that intestinal microorganisms may play a potential role in reducing cardiovascular risk factors. We also discuss the potential of microbiota-targeted therapeutics as novel treatment strategies for the preventing and/or treating CVD, and highlight the need to establish causal links between therapeutics for metabolic disease, gut microbiota modulation, and cardiovascular protection.

\section{THE ROLE OF GUT MICROBIOTA INMETABOLIC DISORDERS AND CARDIOVASCULAR DISEASE}

There are more than 1,000 microbial species in the human gastrointestinal tract, mainly belonging to five phyla (Bacteroidetes, Firmicutes, Actinobacteria, Proteobacteria, and Verrucomicrobia). Of these, anaerobic Bacteroidetes and Firmicutes contribute more than $90 \%$ of all bacterial species (Qin et al., 2010). They constitute $90 \%$ of the total number of cells contained within our bodies, and the number of microbial genes is over 100 times larger than the human nuclear genome (Grice and Segre, 2012; Hillman et al., 2017). Gene sequencing data have indicated that the gut metagenome is involved in host physiology and pathophysiology processes such as the digestion and absorption of nutrients, stimulation of the immune system, and regulation of host metabolic pathways (Costello et al., 2012; Nicholson et al., 2012; Palm et al., 2015). Harmful alterations in the gut microbiota composition, a condition referred to as dysbiosis, have been associated with the development of metabolic disorders. For instance, it is generally believed that a rise in the ratio of Firmicutes to Bacteroidetes is related to a chronic low-grade inflammatory status and an elevated capability for obtaining more energy from food (Pahwa et al., 2017). The diversity of gut microbiota in obese patients is visibly lower than that in the normal weight population (Wolf, 2006) and this decrease may lead to a higher insulin resistance (Jiao et al., 2018). In addition, there is a link between the gut microbiota and different kinds of CVD. The use of broad-spectrum antibiotics affects analytes produced during the catabolism of aromatic amino acids with an associated reduction in myocardial infarct size (Lam et al., 2016). A characteristic change in gut microbiota has been observed in coronary artery disease patients in which Lactobacillales increased and phylum Bacteroidetes (Bacteroides and Prevotella) decreased; this was not observed in a comparative cohort of patients with diabetes (Emoto et al., 2016). In another metagenome-wide association study, an increased abundance of Enterobacteriaceae and Streptococcus spp. and a relatively depleted abundance of butyrate-producing bacteria was observed in patients with atherosclerotic CVD compared to healthy controls (Jie et al., 2017).

Although the unified mechanism behind this kind of correlation has not yet been established, there are still some possible explanations for the interplay between gut microbiota and the host. In some cases, structural components of gut microbiota such as lipopolysaccharides (LPS) and peptidoglycans can be used as signaling molecules; these can be recognized as pattern recognition receptors, such as toll-like receptors (TLRs) and nucleotide oligomerization domain-containing receptors (Curtiss and Tobias, 2009; Philpott et al., 2014), which can stimulate and instruct host immune response both at the epithelial cell border as well as within the vasculature (Medzhitov, 2007). When gut wall barrier function is impaired, low levels of gut-derived bacteria can actually enter the bloodstream to elicit a chronic low grade pro-inflammatory and pro-oxidative stress status that is commonly referred to as "metabolic endotoxemia" which may result in several cardiovascular risk factors, such as obesity, insulin resistance, dyslipidemia, and oxidative stress (Neves et al., 2013).

Gut microbiota can also produce various metabolites that can act locally in the gut as well as travel systemically to directly or indirectly affect distal organs. Here, we provide a brief introduction to some main classes of gut microorganismdependent metabolites that have been linked to CVD risk in either humans or mouse models.

Short-chain fatty acids (SCFAs) are products of the fermentation of dietary fibers by gut bacteria (Topping and Clifton, 2001). A growing body of evidence suggests that SCFAs, as metabolic targets, have a potential role in preventing and counteracting obesity and obesity-related diseases (impaired glucose metabolism and insulin resistance) (Canfora et al., 2015; Koh et al., 2016). By regulating the release of intestinal hormones, such as peptide YY and glucagon-like peptide-1 (GLP-1), SCFAs can suppress appetite, increase energy expenditure, and prevent diet-induced obesity (Gao et al., 2009; Lin et al., 2012; Chambers et al., 2015). Therefore, it may be possible to show that SCFAs are involved in cardiovascular physiological processes mainly by 
establishing links with cardiovascular risk factors (Canfora et al., 2015; Koh et al., 2016).

Bile acids (BAs) are an important group of metabolites with a profound effect on human health and microbiota are involved in the bio-transformation of BAs. For instance, bile acid deconjugation is carried out by bacteria with bile salt hydrolase (BSH) activity, this prevents the active absorption of BAs in small intestine via the apical sodium-dependent bile acid transporter (ASBT) (Wahlstrom et al., 2016). Besides, gut microbiota are the sole source of $7 \alpha$ and $7 \beta$ dehydrogenase activity, which generates "secondary" bile acids (SBAs) such as deoxycholic acid (DCA) and lithocholic acid (LCA) (Wahlstrom et al., 2016). These bacterial modified bile acids regulate a wide range of metabolic and immune-related processes by acting as endocrine-like signaling molecules and engaging multiple bile acid receptors like farnesoid $\mathrm{X}$ receptor (FXR), pregnane $\mathrm{X}$ receptor (PXR), and $\mathrm{G}$ protein-coupled bile acid receptor (TGR5) (Wahlstrom et al., 2016; Joyce and Gahan, 2017; Brown and Hazen, 2018; Schramm, 2018). Importantly, studies have shown that the deficiency of either FXR or PXR in mice can cause reduced atherosclerosis, whereas TGR5 knockout mice are protected against atherosclerosis development (Zhang et al., 2006; Pols et al., 2011; Sui et al., 2011).

Trimethylamine oxide (TMAO) has gained considerable attention in recent years as a biomarker for human CVD risk and a promoter of atherothrombotic diseases (Wang et al., 2011; Zhu et al., 2016). After high-fat food intake, several distinct gut microbial enzyme complexes can metabolize nutrients such as phosphatidylcholine and choline to generate the primary gut microbial metabolite trimethylamine, which is subsequently converted by the host flavin monooxygenase enzyme family to TMAO in the liver (Bennett et al., 2013). TMAO then increases atherosclerotic CVD, including major adverse cardiovascular events (MACE) (death, myocardial infarction, and stroke) by altering cholesterol transport, modulating platelet hyperresponsiveness, and increasing macrophage activation (Tang et al., 2013; Koeth et al., 2014; Wang et al., 2015; Zhu et al., 2016).

In addition, bacterial metabolites originating from amino acids also play an important role in host physiology. For example, indole (a tryptophan metabolite) can be produced by many bacterial species including Escherichia coli, Clostridium spp., and Bacteroides spp. and has been shown to induce the release of GLP-1 in enteroendocrine L-cells. As signaling molecules, indole and some of its derivatives, such as indolepropionic acid (IPA) and indoleacrylic acid (IA) can affect mucosal homeostasis by decreasing intestinal permeability; this is possibly mediated by the PXR (Roager and Licht, 2018). Furthermore, IPA and IA also have anti-oxidative and anti-inflammatory effects (Karbownik et al., 2006; Wlodarska et al., 2017). A recent study also found that phenylacetylglutamine (PAGln), a new CVDpromoting gut microbiota-dependent metabolite originating from phenylalanine, increases the potential for thrombosis via Gprotein-coupled receptors, leading to an increased risk of CVD and MACE (Nemet et al., 2020).

Collectively, both the structural components and metabolites of gut microbiota can be sensed by the host receptor system as signaling molecules, thus establishing direct or indirect interactions with the host. When dysbiosis occurs, many downstream signaling pathways are triggered, resulting in metabolic disorders, and indirectly or directly promoting CVD.

\section{THE EFFECT OF ORAL HYPOGLYCEMIC DRUGS ON GUT MICROBIOTA}

\section{Metformin}

As the first-line orally administered drug for T2DM treatment, metformin is one of the most prescribed compounds on the market, mainly due to its safety profile, proven efficacy, and low cost, as well as its high clinical value in reducing the incidence of cardiovascular events and mortality rates (UKPDS Group, 1998; Morgan et al., 2014). As far back as 1998, the UK Protective Diabetes Study (UKPDS) found that, compared to conventional treatment with diet alone, metformin treatment led to a lower all-cause mortality and a concurrent lower risk in the incidence of myocardial infarction in newly diagnosed T2DM after a median follow-up of 10.7 year (UKPDS Group, 1998). At the time, this phenomenon was explained as the beneficial effect of tight glycemic control from metformin that prevented future cardiovascular consequences. However, in a subsequent follow-up study 10 years after UKPDS, a continuous risk reduction of myocardial infarction was found in patients treated with metformin despite there being no changes in glycated hemoglobin (HbA1c) levels (Holman et al., 2008). Similar results have also been observed in subsequent clinical studies (Kooy et al., 2009; Hong et al., 2013; Han et al., 2019), which suggested the pleiotropic effects of metformin in the heart and blood vessels, independent of its glucose-lowering activity.

At present, the multiple action mechanisms of metformin are still debated, and the hypoglycemic effect has been primarily ascribed to its capacity to inhibit hepatic gluconeogenesis. However, despite the high accumulation of metformin in the intestinal wall, concentration in the plasma is up to 300 times lower (Bailey et al., 2008). In addition, intravenous administration of metformin does not improve glycemia (Bonora et al., 1984; Stepensky et al., 2002). This suggests that the intestine is possibly the main target organ of metformin action (Duca et al., 2015; Buse et al., 2016; Brunkwall and Orho-Melander, 2017). It is known that metformin can increase peripheral glucose uptake and modulate the incretin pathway by improving GLP1 receptor expression in the pancreatic islets and increasing plasma levels of GLP-1 (Viollet et al., 2012; Pernicova and Korbonits, 2014; Montandon and Jornayvaz, 2017). GLP-1 is a major metabolic hormone produced by enteroendocrine $\mathrm{L}$ cells. Not only can GLP-1 lower blood glucose by inducing insulin secretion, inhibiting glucagon release, and slowing gastric emptying, it also instigates many cardioprotective pathways, possibly contributing to the cardiovascular protection observed in metformin users (Monami et al., 2017). The results of a human clinical study also provide consistent evidence for the intestine hypothesis. The delivery of delayed-release metformin with $50 \%$ of the bioavailability of extended-release metformin to the lower bowel results in significant glucose-lowering efficacy but with 
lower doses and significantly lower systemic exposure than that of extended-release metformin (Foretz et al., 2019). Although very little is currently known about the bacterial targets of metformin, there is convincing evidence that metformin can reshape the composition of human gut microbiota.

The abundance of Akkermansia muciniphila, a mucindegarding bacterium, is reduced in individuals with obesity and diabetes compared to healthy individuals, as well as in rodents (Everard et al., 2013; Dao et al., 2016; Plovier et al., 2017). Furthermore, a higher abundance is associated with a healthier metabolic status and improvements in the cardiac metabolic parameters in individuals with obesity (Dao et al., 2016). Participants with diabetes that were also taking metformin had a higher relative abundance of this microbe compared to participants with diabetes that were not taking metformin and participants without diabetes (de la Cuesta-Zuluaga et al., 2017; Cani, 2018). This is in agreement with other in vitro experiments showing that metformin directedly increases the growth of A.muciniphila (Wu et al., 2017). As Akkermansia uses mucus as a nutrient source, Metformin is further found to increase the number of goblet cells. Goblet cells produce gastrointestinal mucins that protect the underlying epithelium from pathogens, and the number of it was positively correlated with the abundance of Akkermansia (Shin et al., 2014). In addition, one study found that a recombinant protein isolated by $A$. muciniphila can improve the intestinal barrier through interaction with TLR2 (Plovier et al., 2017), even A. muciniphila derived extracellular vesicles can influence gut permeability through the regulation of tight junctions (Chelakkot et al., 2018). Decreased gut permeability limits the release of endotoxins such as LPS, which in turn improves the chronic low-grade inflammatory status and reduces vascular inflammation (Tang et al., 2019). A recent study showed that fecal microbiota transplantation (FMT) using fecal material from metformintreated mice can not only upregulate the expression of GLP-1 and pattern-recognition receptors TLR1 and TLR4 to improve hyperglycemia caused by a high-fat diet (HFD), but can also downregulate the expression of the inflammatory cytokine IL-18 (Lee et al., 2019) which has been identified as a possible risk factor for CVD (Jefferis et al., 2011). However, metformin treatment was recently found to be associated with an increased concentration of TMAO (a promoter of atherothrombotic disease) (Brown and Hazen, 2018; Croyal et al., 2020). Further, results from another animal study suggested that the plasma TMAO level were significantly associated with the relative abundance of Akkermansia within the jejunum and cecum (Koeth et al., 2014). Therefore, it is difficult to comprehensively determine whether increased Akkermansia abundance is ultimately beneficial for cardiovascular disease.

Another therapeutic effect of metformin is its influence on the interactions between BAs and the microbiota. The inhibitory effect of biguanides on bile acid absorption has been acknowledged since the 1970s (Caspary and Creutzfeldt, 1975). Metformin can reduce active bile acid absorption via ASBT inhibition (Napolitano et al., 2014). Subsequent modifications of bile acid-mediated activation of the nuclear FXR and the cell surface TGR5 might influence GLP-1 secretion from L cells (Bronden et al., 2017; van Stee et al., 2018). In a recent study,
Sun et al. (2018) performed metagenomic and metabolomic analyses of samples from individuals with newly diagnosed T2DM after 3 days of metformin treatment. The BSH activity of Bacteroides fragilis was strongly reduced after metformin administration, and glycoursodeoxycholic acid (GUDCA) was increased in the gut. They further confirmed that GUDCA is an intestinal FXR antagonist that controls bile acid homeostasis and glycolipid metabolism (Matsubara et al., 2013). Thus, they concluded that metformin acts in part through a $B$. fragilis GUDCA-intestinal FXR axis to improve metabolic dysfunction (Sun et al., 2018). In addition, B. fragilis is involved in the transformation of primary bile acids to SBAs such as LCA, which can activate the PXR (Staudinger et al., 2001). Considering the possible correlation between FXR, PXR and atherosclerosis (Zhang et al., 2006; Sui et al., 2011), the cardioprotective effect of metformin may be related to its regulation of $B$. fragilis. Moreover, levels of Firmicutes (e.g., Clostridium perfringens) have been shown to decrease in metformin-treated patients (Karlsson et al., 2013), which in turn can alter bile acid metabolism by reducing the circulating levels of pro-inflammatory bile acids (DCA and TDCA) (Fujisaka et al., 2018).

Metformin can also increase the abundance of SCFAproducing taxa and species (e.g., Butyrivibrio, Bifidobacterium bifidum, and Megasphaera) (Forslund et al., 2015; de la CuestaZuluaga et al., 2017). As mentioned earlier, SCFAs function as a macronutrient energy source and hormone-like signaling molecules that enter the portal circulation to signal through two orphan G protein-coupled receptors, GPR41 (Samuel et al., 2008) and GPR43 (Kimura et al., 2013), and mediated AMPK activation in diverse tissues to regulate innate immunity and host metabolism (den Besten et al., 2015; Brown and Hazen, 2018). In addition, metformin can increase the enrichment of Lactobacillus and Bifidobacterium (Wu et al., 2017). Whereas Lactobacillius promote GLP-1 release from L-cells by increasing the apical expression of sodium glucose cotransporter-1 (SGLT1) (Bauer et al., 2018), Bifidobacterium have also proven to be negatively related to $\mathrm{HbAlc}$.

Although the intestine hypothesis of the metformin action mechanism provides an explanation for its cardiovascular benefits, this still requires further validation, especially regarding the role of metformin in non-diabetic patients with CVD. Limited by indications, there has been very limited research on this subject. A small randomized double-blind placebocontrolled study consisting of 33 non-diabetic women showed that metformin can improve vascular function and reduce myocardial ischemia in female patients with angina compared to a placebo (Jadhav et al., 2006). In addition, the recent proofof-concept MET-REMODEL trial (Metformin and its Effects on Left Ventricular Hypertrophy in Normotensive Patients With Coronary Artery Disease) in patients with coronary artery disease (CAD), insulin resistance (IR), and/or pre-diabetes found a beneficial effect of metformin on left ventricular mass indexed to height (LVMI), LVM, office systolic blood pressure, and oxidative stress (Mohan et al., 2019). However, some pilot clinical studies with other surrogate end-points for cardiovascular events such as carotid intimal media thickness have not yielded positive results (Preiss et al., 2014; Kulkarni et al., 2018; Rena and Lang, 2018). Thus, further evidence is needed before 
metformin can be recommended for non-diabetic patients with CVD.

\section{Alpha- Glucosidase Inhibitors}

Accumulating evidence suggests that post-prandial hyperglycemia (PPHG) is a powerful predictor of diabetic cardiovascular events (DECODE Study Group, 2001; Ning et al., 2010). Studies have found that PPHG can increase oxidative stress through endothelial dysfunction (Kawano et al., 1999) and low-grade chronic inflammation (Esposito et al., 2002), leading to the development of atherosclerosis and the occurrence of cardiovascular events (Node and Inoue, 2009). Alpha-glucosidase inhibitors ( $\alpha$-GIs) are microorganism-derived anti-glycemic drugs that have been proven to reduce PPHG and are associated with a favorable impact on an array of CVD surrogate markers (Geng et al., 2011; Standl et al., 2014).

The classic mechanism of $\alpha$-GIs is believed to act through the inhibition of the host glucoamylase, delaying carbohydrate digestion and pushing the carbohydrates to the lower intestinal tract, where they can be transformed into the food for the intestinal bacterial community. This makes it possible to change the gut microbiota composition. Although the specific mechanism of $\alpha$-GIs that regulates the gut microbiota is unclear, there is still evidence that apart from reducing post-prandial blood glucose, AGIs can also protect the cardiovascular system by reshaping the gut microbiota composition.

Acarbose, which is derived from the fermentation process of Actinoplanes utahensis, is the most widely used $\alpha$-GI in hyperglycemia treatment (Weng et al., 2015). Previous studies suggest that acarbose treatment can reduce the risk of cardiovascular endpoints in patients with diabetes (Hanefeld et al., 2004; Hanefeld, 2007; Chen et al., 2014). A clinical study involving 106 patients with newly diagnosed T2DM found that acarbose treatment can significantly change the gut microbiota composition compared to glipizide, especially increasing the abundance of species possessing high BSH activity, such as Lactobacillus gasseri and Bifidobacterium longum, while depleting putrefactive species of Bacteroides, Alistipes and Clostridium, thereby changing the relative abundance of microbial genes involved in BA metabolism and contributing to beneficial effects on the host metabolism (Gu et al., 2017). Another study confirmed this result (Su et al., 2015), which also found that acarbose can reduce the level of LPS in the blood by increasing the number of Bifidobacterium and reducing the number of Enterococcus faecalis (Kikuchi et al., 2018), and thus, alleviate chronic low-level inflammation in patients. These results indicate that changes in the gut microbiota after acarbose treatment can bring greater cardiovascular benefits to diabetic patients. In addition, the cardiovascular benefits of acarbose can be explained by new insights into the production of SCFAs and the release of $\mathrm{H}_{2}$ gas by the gut microbiota. Increasing evidence supports the view that the use of acarbose increases the number of colonic bacteria, which in turn promotes the production of SCFAs and $\mathrm{H}_{2}$ gas through the fermentation of undigested carbohydrates in the lower digestive tract (Suzuki et al., 2009; Hai-xia et al., 2011). As mentioned previously, SCFAs can signal through specialized host receptor systems to regulate innate immunity and host metabolism. $\mathrm{H}_{2}$ gas can be absorbed from the intestine into the circulation to mediate the inhibition of pro-inflammatory cytokines, especially IL- $1 \beta$, TNF- $\alpha$, and IL- 6 in inflammatory tissues. Moreover, a recent study proposed that $\mathrm{H}_{2}$ converts the quinone intermediates into the fully reduced ubiquinol, thereby increasing the antioxidant capacity of the quinone pool and preventing the generation of reactive oxygen species (Ishibashi, 2019). Clinical evidence and experimental results strongly suggest that reactive oxygen species are the main cause of cardiovascular diseases such as hypertension, atherosclerosis, angina pectoris, myocardial infarction, and heart failure (Touyz, 2004; Madamanchi et al., 2005).

Although current microbiota studies support acarbose for its cardiovascular benefits, its cardioprotective effect in non-diabetic patients is still controversial. The STOP-NIDDM trial (Study to Prevent Non-Insulin-Dependent Diabetes Mellitus) showed that decreasing PPHG with acarbose was not only associated with a reduction in the incidence of new-onset diabetes as the primary objective of this study, but also with a relative risk reduction in the development of cardiovascular events, especially in the risk of myocardial infarction as assessed by a planned post hoc analysis of predefined secondary outcomes (Chiasson et al., 2003). However, the recently completed ACE (Acarbose Cardiovascular Evaluation) trial on patients with coronary heart disease and impaired glucose tolerance showed that acarbose delayed progression to type 2 diabetes, but was neutral with respect to MACE (Holman et al., 2017).

\section{GLP-1 Receptor Agonists}

As mentioned earlier, GLP-1 is an insulinotropic hormone secreted by gastrointestinal neuroendocrine cells. The body can help to reduce blood sugar by secreting GLP-1 after eating, but natural GLP-1 is rapidly degraded by DPP-4 secreted in the intestine. Due to the relative lack of insulin or insulin resistance, diabetes patients have high blood glucose levels. At this time, auxiliary substances such as GLP-1 are particularly important. GLP-1 receptor agonists (GLP-1RAs), a new type of hypoglycemic drug, can not only increase insulin secretion, suppress appetite, and control weight by activating GLP-1 receptors (Vilsboll et al., 2012; McAdam-Marx et al., 2014), they can also control hyperglycemia by inhibiting glucagon secreted by islet $\alpha$ cells (Meier, 2012). Of these, liraglutide (Marso et al., 2016b) and semaglutide (Marso et al., 2016a) have also been found to have cardioprotective effects. Interestingly, although all GLP-1RAs currently on the market are subcutaneous injections, liraglutide can change the composition of microorganism community (Zhang et al., 2018; Madsen et al., 2019). This may be due to the GLP-1 level because it influences the gut transit time and gastric emptying rate, and could therefore modify the gut lumen internal environment (local $\mathrm{pH}$ value and nutrient composition).

Zhang et al. (2018) found that SCFA-producing bacteria including Bacteroides, Lachnospiraceae, and probiotic Bifidobacterium, arec selectively enhanced in liraglutide-treated diabetic male rats. SCFA can affect intestinal anti-inflammatory abilities (Boulange et al., 2016) and prevent a low-grade inflammatory response. In addition, Lachnospiraceae exhibit 
a positive relationship with peak oxygen uptake, the gold standard measure of cardiorespiratory fitness (Estaki et al., 2016). Fuerthermore, liraglutide can reduce obesity-associated bacteria (Erysipelotrichaceae, Marvinbryantia, Roseburia, Candidatus Arthromitus, Parabacteroides, Romboutsia, and Ruminiclostridium) in both obese and diabetic obese rats (Wang et al., 2016; Zhao et al., 2018) and increased the lean-related phylotypes such as Prevotella, Blautia and Coprococcus. The Akkermansia genus was significantly reduced in patients with a long T2DM duration. After comparing the gut microbiota of subjects receiving GLP-1 RA and metformin, a higher Akkermansia abundance was found in the liraglutide-treated patients (Wang et al., 2018b). As mentioned earlier, a higher abundance of Akkermansia is beneficial for metabolism and the gut barrier function, it is also related to the plasma concentration of TMAO. However, it is still unknown whether liraglutide treatment can result in increased TMAO concentrations.

It has been found that GLP-1 RA can reduce the formation of atherosclerosis by improving vascular endothelial function, inhibiting inflammatory response, and reducing myocardial infarction ischemia, thereby preventing diabetic vascular disease (Khat and Husain, 2018). Although there are few pilot clinical studies on using GLP-1RA to prevent CVD, the regulation of gut microbiota may also become one of the potential cardiovascular benefit mechanisms of GLP-1RA in the future.

\section{Sodium Glucose Co-transporter 2 Inhibitors (SGLT2i)}

SGLT2i are a new class of oral hypoglycemic drugs. The action mechanism of SGLT2i mainly promotes urine glucose excretion by inhibiting the reabsorption of sodium glucose in the proximal renal tubules. Due to its unique glucose excretion mechanism, the hypoglycemic effect of SGLT2 inhibitors is unaffected by the function of islet cells and insulin resistance, thus reducing the risk of hypoglycemia (Whalen et al., 2015). Recently, as the benefits of SGLT-2i in cardiovascular outcome trials (COVTs) outweigh those of other anti-diabetic drugs (Zinman et al., 2016; Neal et al., 2017; Wiviott et al., 2019), they have received increasing attention in the field of diabetes. Three CVOTs on empagliflozin, canagliflozin, and dapagliflozin in T2DM patients and either established CVD or multiple cardiovascular risk factors showed remarkable positive results and proved the significant role of these three types of SGLT2i in reducing MACE, cardiovascular mortality, all-cause mortality, and hospitalization for heart failure (Zinman et al., 2016; Neal et al., 2017; Wiviott et al., 2019).

Based on our current understanding, the cardiovascular protection mechanism of SGLT2i mainly lies in their ability to reduce cardiac inflammation, oxidative stress, cell apoptosis, mitochondrial dysfunction, and ionic abnormalities (Lahnwong et al., 2018). As of yet, consistent evidence for their effect on microbiota is lacking. A pharmacological experimental study of a dual SGLT1/2 inhibitor complex showed that the relative abundance of both the bacterial orders and bacteria of interest in metabolic disease (e.g., Akkermansia spp.) in C57BL/6 mice was unaltered after drug treatment $(1 \mathrm{mg} / \mathrm{kg}$ p.o. for 6 consecutive days) (Lahnwong et al., 2018). Another animal experiment for dapagliflozin (dapa) (Lee et al., 2018) found that treatment with dapa had little effect on the gut microbota of control mice, but did subtly change the richness and diversity of the microbial community in diabetic $\mathrm{Db}$ mice. On the one hand, the Firmicutes to Bacteroidetes ratio in the $\mathrm{Db}+$ dapa group was reduced, which suggests that dapa may reduce the level of inflammation in the body by adjusting the gut microbiota. The experiment also found that at the species level, A. muciniphila showed a tendency to increase in the $\mathrm{Db}+$ dapa group compared to the $\mathrm{Db}$ group. A. muciniphila has been shown to improve metabolic outcomes, including vascular function (Qin et al., 2012; Li et al., 2016), while participating in the protection of the intestinal mucosal barrier and controlling low-grade inflammation (Chelakkot et al., 2018; Lee et al., 2019; Tang et al., 2019).

\section{Dipeptidyl Peptidase-4 Inhibitors (DPP-4i)}

DPP-4i are an extensively used class of oral hypoglycemic agents that target the DPP-4 enzyme and inhibit the degradation of GLP-1 to reduce blood glucose levels (Holst and Deacon, 1998). Although the existing CVOTs with DPP-4i showed no difference in combined MACE outcomes (Scirica et al., 2013; White et al., 2013; Green et al., 2015), DPP-4i may also positively influence surrogate vascular end points and other cardiovascular risk factors, as extensively discussed in previous reviews (Mulvihill and Drucker, 2014; Scheen, 2018). In parallel, some progress has been made in identifying the influence of DPP-4i on gut microbiota, although most of the evidence comes from animal experiments.

Currently, DPP-4i studies, especially those on sitagliptin and vildagliptin, have shown similar evidence that DPP-4i treatment partially reverses HFD-induced dysbiosis, significantly reduces the Firmicutes to Bacteroidetes ratio, and alters the population of SCFA-producing bacteria. Vildagliptin also has a potential effect on inflammation due to the reduction of TLR and cytokine expression (Yan et al., 2016; Zhang et al., 2017). All these changes ultimately mediate the beneficial effects of DPP-4i on the host, especially in terms of glucose homeostasis. Another animal study found that both sitagliptin and saxagliptin alter gut microbial composition and promote a functional shift in the gut microbiome. In particular, succinate production is especially increased. Succinate is reportedly a key substrate of intestinal gluconeogenesis in the improvement of glucose metabolism (de Vadder et al., 2016). And GLP-1 is not the main mediator of the effect on gut microbiota, a suggestion that is further supported by the findings of Olivares et al., who proposed the existence of significant intrinsic DPP-4-like activity in gut microbial populations (Olivares et al., 2018). Moreover, by transferring fecal samples from sitagliptin-treated T2DM patients to HFD-fed germ-free mice, this study further demonstrated that the altered microbiome contributes to the hypoglycemic effects of sitagliptin, even in the absence of additional treatments (Liao et al., 2019).

\section{THE EFFECT OF STATIN ON GUT MICROBIOTA}

Hyperlipidemia is an important risk factor for CVD (Isomaa et al., 2001) and is one of the main results of multiple metabolic disorders. Statins, as the most widely used drugs for treating hyperlipidemia, have been recommended by the 
American College of Cardiology/American Heart Association (ACC/AHA) as first-line treatment for hyperlipidemia (Stone et al., 2014). Lipid metabolism regulation is mainly achieved by inhibiting 3-hydroxy-3-methylglutaryl coenzyme-A reductase in the cholesterol synthesis pathway (Stancu and Sima, 2001). Although the efficacy of statins has been proven, their therapeutic effects vary from person to person. A metaanalysis of a statin intervention trial involving 32,258 patients in 37 clinical trials showed that the standard deviation of low-density lipoprotein cholesterol (LDL-C) reduction for all statins and doses ranged from 12.8 to $17.9 \%$ (Karlson et al., 2016). The percentage of patients experiencing a suboptimal response ( $<30 \%$ reduction in LDL-C) ranged from 5.3 to $53.3 \%$. The change was not related to the specific statin dose, but was probably related to the composition of the gut microbiota. In an observational study involving 64 patients with hyperlipidemia (Liu et al., 2018), after 4-8 weeks of rosuvastatin treatment, there was a difference in the microbiota composition of the two groups that showed the best (group I) or suboptimal (group II) clinical results. In particular, the higher abundance of Firmicutes, butyrate-producing bacteria families of Ruminococcaceae, Lachnospiraceae, and Clostridiaceae, and the lower abundance of Bacteroidetes are believed to be related to the best therapeutic effect of rosuvastatin. However, considering the fact that the study lacked a control group and did not sequence the bacteria in either group before treatment, microbiome changes could not be assessed. In another experiment with atorvastatin in rats (Khan et al., 2018a), atorvastatin-treated HFD groups showed a relative increase in biodiversity compared to the HFD control group, and promoted the relative abundance of Proteobacteria, and reduced the abundance of Firmicutes. At the same time, several specific dominant groups including Oscillospira, Parabacteroides, Ruminococcus, unclassified CF231, YRC22 (Paraprevotellaceae), and SMB53 (Clostridiaceae) were observed to have a reversed abundance relative to the HFD group, which was similar to that of the normal rat food control group. In a cross-sectional study, the gut microbiome of 15 untreated hypercholesterolemia patients, 27 atorvastatin-treated hypercholesterolemia patients, with 19 healthy subjects were compared (Khan et al., 2018b). A distinct bacterial signature with species associated with atherosclerosis and inflammation (e.g., Collinsella, Streptococcus) was found in the untreated hypercholesterolemia patients, while an increased abundance of putative anti-inflammatory species (Akermansia muciniphila, Faecalibacterium prausnitzii, and genus Oscillospira) was found in atorvastatin-treated patients.

To investigate the role of gut microbiota in the effect of statins on lowering blood lipid, Wang et al. (2018a) used antibiotics to establish a rat model of microbiome imbalance that lasted for 2 weeks. They found that the abundance of Lactobacillus and Bifidobacterium was remarkably diminished upon antibiotic treatment in the antibiotic+rosuvastatin-treated group compared to that of the rosuvastatin-treated group and control group. Correspondingly, the efficacy of rosuvastatin in lowering the blood levels of total cholesterol and LDLC was significantly compromised. Studies have shown that the presence of Bifidobacterium and Lactobacillus can lead to the alteration of TNF $\alpha$ and IL- 6 levels, which results in the reduction of cholesterol (Veiga et al., 2005). The expression of a cellular transporter of rosuvastatin (OATP1B1) is regulated by a variety of factors including TNF $\alpha$ and IL6 (Le Vee et al., 2009); therefore, it can be speculated that beneficial gut bacteria mediate the lipid-lowering effect of rosuvastatin by influencing the expression of inflammatory factors. In a recent study, Kim et al. (2019) found that atorvastatin and rosuvastatin significantly increased the abundance of the genera Bacteroides, Butyricimonas, and Mucispirillum in elderly obese mice. This change in gut microbiota plays an important role in downregulating IL- $1 \beta$ expression and upregulating TGF $\beta$ expression (Winer et al., 2016; Goncalves et al., 2018) (Figure 1).

In addition to the impact of statin on gut microbiota, there is also evidence that the efficacy of statin medications is regulated by gut microbiota. A study involving plasma metabolomic profiling in 100 human subjects found that baseline concentrations of three secondary bacterial-derived BAs (LCA, taurolithocholic acid, and glycolithocholic acid), positively predicted the magnitude of simvastatin-induced LDLC lowering (Kaddurah-Daouk et al., 2011). An animal study also reported that simvastatin treatment increased the abundance of Bacteroidetes, reduced Firmicutes and Actinobacteria, as well as altering the serum metabolic and bile acids profiles of HFD feeding mice. Moreover, it found that gut microbiota modulation resulting from oral antibiotics attenuated the hypolipidemic effect of simvastatin and suggested that this might be related to the suppression on hepatic CYP7A1, CYP7B1, and FXR proteins that regulate bile acids synthesis from cholesterol by gut microbiota modulation (He et al., 2017).

\section{DISCUSSION}

With the development of gene sequencing technology and human microbiome research, an increasing amount of evidence from animal and human studies has revealed that the activity of gut microbiota is closely related to the occurrence and development of metabolic and CVD. In parallel, gut microbiota have been recognized as playing an important role in the digestion, absorption, metabolism and even the effect of drugs. Therefore, with an in-depth understanding of gut microbiota, it is possible that microbial pharmacology may become an important part of modern pharmacology.

In this review, we summarize the advances of research on several drugs for metabolic diseases with proven cardiovascular benefits in regulating gut microbiota. This enables the identification of microbial targets that may help these drugs to provide their cardiovascular protective effects. At present, the potential benefits of these drugs in reducing CVD risks mainly include the reduction of multiple cardiovascular risk factors such as hyperglycemia, hypertension and hyperlipidemia, as well as other beneficial effects, including increased insulin sensitivity, weight loss, reduced of oxidative stress and lowgrade inflammation, restored of endothelial function, and the inhibition of key platelet activation pathways (Standl et al., 2014; Boyle et al., 2018; Lahnwong et al., 2018; Zilov et al., 2019). 

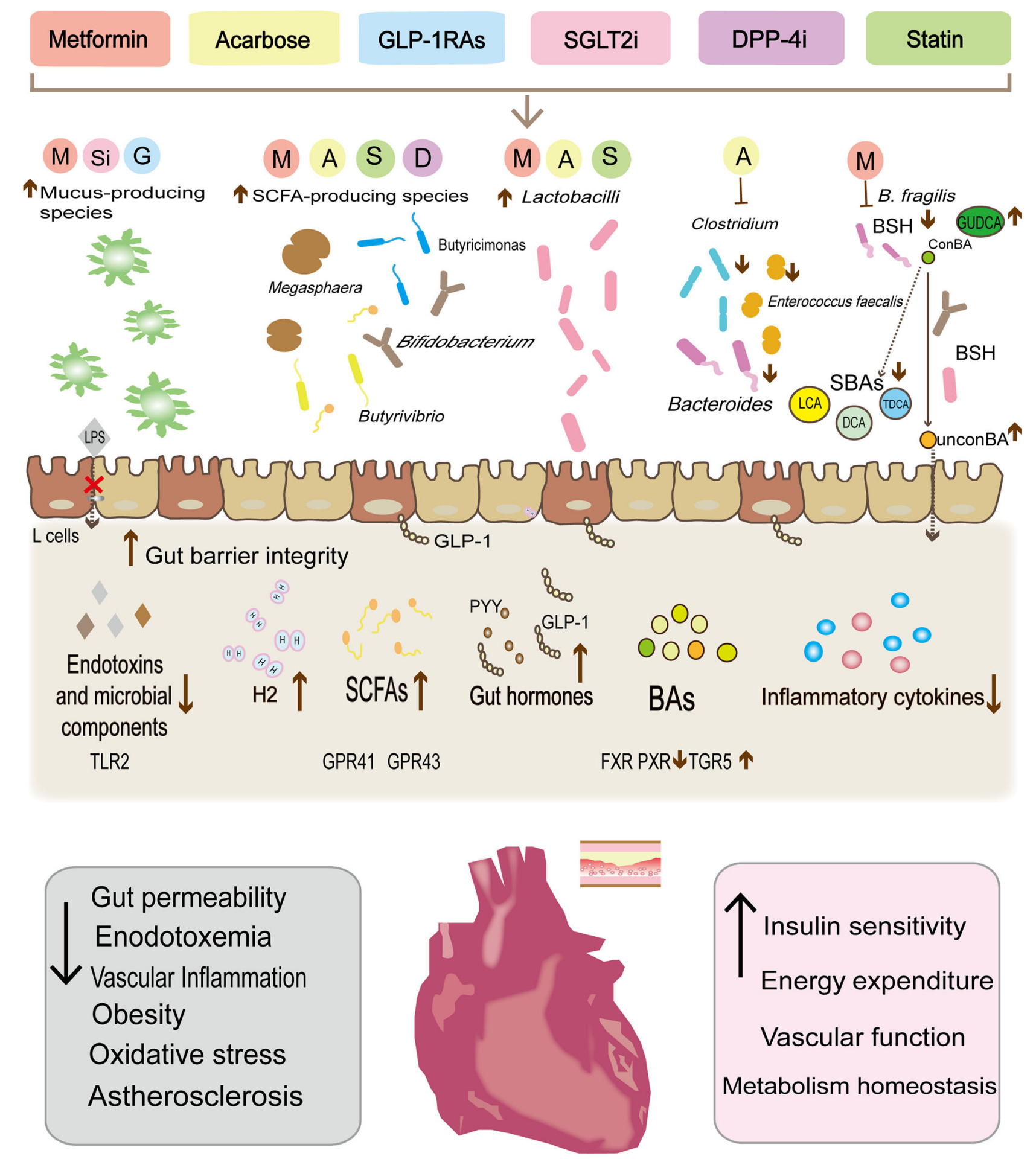

FIGURE 1 | Metabolic therapy drugs reshape the gut microbiota composition, modulate the abundance of specific gut bacteria, and then control the release of the microbial derivative to achieve cardiovascular protection and reduce cardiovascular risk factors. Metformin, liraglutide, and dapalizine have been found to increase the abundance of Akkermansia muciniphil, which can enhance the function of the intestinal barrier, limit endotoxin leakage. In parallel, the increase in the abundance of some species (Bacteroides, Butyricimonas, Lactobacilli, and Mucispirillum) after drug treatment has also been found to be significantly related to the decrease in secretion of pro-inflammatory cytokines (IL-18, IL-1 $\beta$, IL-16, and TNF $\alpha$ ). In turn, the decrease of the low-level inflammatory status has been shown to increase insulin sensitivity and benefit the vascular function. Similarly, both metformin and acarbose can reduce Bacteroides and Clostridium clusters, and may alter bile acid metabolism, with a reduction of bile acid reabsorption and secondary bile acids, thereby influencing the excitability of various atherosclerosis-related receptors (PXR, FXR, and TGR5) and stimulating GLP-1 secretion. Moreover, metformin, acarbose, liraglutide, rosuvastatin, and atorvastatin have also been found to increase 
FIGURE 1 | SCFAs-producing species (Butyrivibrio, Bifidobacterium, Megaspheara, and Butyricimonas Lactobacilli et al.). SCFAs, through the G protein-coupled receptors GPR-41 and GPR43 mediated AMPK activation in diverse tissues to regulate innate immunity and host metabolism. In addition, Lactobacilli can also promote GLP-1 secretion by increasing the apical expression of SGLT1 cotransporter. Finally, acarbose increases the release of $\mathrm{H}_{2}$ gas by the gut microbiota as well as preventing oxygenated stress damage.

The effects of these drugs on the gut microbiota could be one explanation for most of these benefits.

Despite some exciting findings, few studies provide causal evidence that drugs are directly involved in cardiovascular protection through the gut microbiota. On the one hand, gut microbiota vary greatly from individual to individual. The genetic background, lifestyle, emotional state, and method of childbirth of the host all have a great influence on gut microbiota composition (Wen and Duffy, 2017). On the other hand, gut microbiota are not homogenous along the digestive tract, especially between the mucosa (cecum or colon) and stool (Eckburg et al., 2005). Most of the existing studies have selected samples from the cecum, colon, and feces for gene sequencing, and the results are often offset by the location of the selected tissues. Therefore, more study designs using antibiotics or FMT should be encouraged to clarify the causal relationship between microbiome changes and CVD, then to verify these findings through large-sample prospective clinical trials.

Among the drugs we have discussed, there is no doubt that metformin is the most studied. Studies in rodents and humans have generally suggested that metformin can protect the cardiovascular system by increasing SCFA-producing bacteria, interfering with the bioconversion of BAs, and promoting the growth of anti-inflammatory probiotics. Even if the increased TMAO concentration associated with metformin has a negative effect on the cardiovascular system, one may hypothesize that considering the overall effects on the gut microbiota, metfomin treatment could still result in positive cardiovascular outcomes. However, there are still few studies on other drugs mentioned in this manuscript. Current researches speculates that they may achieve regulate immunity, host metabolism, and oxidative stress by promoting the growth of beneficial bacteria and inhibiting the expansion of harmful bacteria, thereby indirectly providing cardiovascular protection. However, many studies have analyzed the gut microbiota at the phylum level. Expressions such as "SCFA-producing bacteria" and "Firmicutes to Bacteroidetes ratio" may be too simple and broad, because these bacteria are diverse, widely distributed and even compete with one another (Becker et al., 2011). There have been many attempts at treatment, such as probiotic supplementation and FMT (Brown and Hazen, 2017), we believe that with the reduction of sequencing costs, metagenomic sequencing will become more and more popular, and the refinement of microbiome structure analysis will help people better understand, screen and utilize the specific functions of microbiota.

In addition, research on the molecular biological mechanisms of drug-microbe interactions and microbe-host interactions are still in progress. Existing studies show that microbial derivatives play an important role in regulating human physiological activities and protecting the cardiovascular system.
These derivatives might include new mediators and potential pharmacological targets for the treatment of cardiometabolic diseases. Non-lethal small-molecule inhibitors of TMAO production can protect mice from diet-enhanced atherosclerosis (Wang et al., 2015). Therefore, additional molecular biology studies on both drug-microbe interactions and microbe-host interactions are still needed. The amount of chemicals and metabolites derived by gut microbiota is staggering. Once a desired microbial derivative is identified, it may become a new drug target for cardiometabolic disease.

\section{CONCLUSION}

The current microbiome studies on antidiabetic agents and lipid lowering agents mainly focus on the role of gut microbiota in regulating metabolic disorders. However, several metabolic therapy drugs have been proven to have additional cardiovascular benefits. Simultaneously, the influence of gut microbiota on the cardiovascular system is being recognized gradually. In this review, we linked the recent advances in microbiome studies on metabolic therapy drugs with their cardiovascular protective effects. Endogenous or exogenous factors change gut microbiota composition, which affect human physiological functions by releasing and regulating a variety of mediators including SBAs, TMAO, SCFAs, inflammatory factors, endotoxins, and intestinal hormones. Existing metabolic therapy drugs may regulate metabolism, immunity, oxidationreduction balance, and other functions by changing the abundance of certain bacteria in the intestinal tract, thus providing cardiovascular protection. However, more detailed experimental and clinical investigations are needed in order to better understand the molecular mechanism of these drugs' regulation of gut microbiota and to identify new targets for microorganism-targeted therapeutics.

\section{AUTHOR CONTRIBUTIONS}

Q-YD and J-XT conceived the work. Q-YD prepared the first draft of the manuscript and designed the figure. X-lT, L-HZ, $\mathrm{ML}$, and F-ML critically revised the manuscript and agreed with the manuscript's results and conclusion. X-XW, LH, Y-JZ, Z-ZG, $\mathrm{H}-\mathrm{YY}$, and X-YF contributed to the writing of the manuscript. All authors critically reviewed and approved the final version of the article.

\section{FUNDING}

This work was partially supported by the Young Elite Scientists Sponsorship Program by CAST (YESS20170034 and 2018QNRC2-C10), the National Natural Science Foundation of China (Nos. 81904187, 81274000, and 81803923), Special 
Scientific Research for Traditional Chinese Medicine of China (201507001-11), Capital Health Development Research Project (CD2020-4-4155), and the Outstanding Young Scientific and Technological Talents Program (ZZ13-YQ-026).

\section{REFERENCES}

Bailey, C. J., Wilcock, C., and Scarpello, J. H. (2008). Metformin and the intestine. Diabetologia 51, 1552-1553. doi: 10.1007/s00125-008-1053-5

Barr, E. L., Zimmet, P. Z., Welborn, T. A., Jolley, D., Magliano, D. J., Dunstan, D. W., et al. (2007). Risk of cardiovascular and all-cause mortality in individuals with diabetes mellitus, impaired fasting glucose, and impaired glucose tolerance: the Australian diabetes, obesity, and lifestyle study (AusDiab). Circulation 116, 151-157. doi: 10.1161/CIRCULATIONAHA.106.685628

Bauer, P. V., Duca, F. A., Waise, T. M. Z., Rasmussen, B. A., Abraham, M. A., Dranse, H. J., et al. (2018). Metformin alters upper small intestinal microbiota that impact a glucose-SGLT1-sensing glucoregulatory pathway. Cell Metab. 27, 101-117.e105. doi: 10.1016/j.cmet.2017.09.019

Becker, N., Kunath, J., Loh, G., and Blaut, M. (2011). Human intestinal microbiota: characterization of a simplified and stable gnotobiotic rat model. Gut Microbes 2, 25-33. doi: 10.4161/gmic.2.1.14651

Bennett, B. J., de Aguiar Vallim, T. Q., Wang, Z., Shih, D. M., Meng, Y., Gregory, J., et al. (2013). Trimethylamine-N-oxide, a metabolite associated with atherosclerosis, exhibits complex genetic and dietary regulation. Cell Metab. 17, 49-60. doi: 10.1016/j.cmet.2012.12.011

Bonora, E., Cigolini, M., Bosello, O., Zancanaro, C., Capretti, L., Zavaroni, I., et al. (1984). Lack of effect of intravenous metformin on plasma concentrations of glucose, insulin, C-peptide, glucagon and growth hormone in nondiabetic subjects. Curr. Med. Res. Opin. 9, 47-51. doi: 10.1185/030079984091 09558

Boulange, C. L., Neves, A. L., Chilloux, J., Nicholson, J. K., and Dumas, M. E. (2016). Impact of the gut microbiota on inflammation, obesity, and metabolic disease. Genome Med. 8:42. doi: 10.1186/s13073-016-0303-2

Boyle, J. G., Livingstone, R., and Petrie, J. R. (2018). Cardiovascular benefits of GLP-1 agonists in type 2 diabetes: a comparative review. Clin. Sci. 132, 1699-1709. doi: 10.1042/CS20171299

Bronden, A., Alber, A., Rohde, U., Rehfeld, J. F., Holst, J. J., Vilsboll, T., et al. (2017). Single-dose metformin enhances bile acid-induced glucagon-like peptide-1 secretion in patients with type 2 diabetes. J. Clin. Endocrinol. Metab. 102, 4153-4162. doi: 10.1210/jc.2017-01091

Brown, J. M., and Hazen, S. L. (2017). Targeting of microbe-derived metabolites to improve human health: the next frontier for drug discovery. J. Biol. Chem. 292, 8560-8568. doi: 10.1074/jbc.R116.765388

Brown, J. M., and Hazen, S. L. (2018). Microbial modulation of cardiovascular disease. Nat. Rev. Microbiol. 16, 171-181. doi: 10.1038/nrmicro.2017.149

Brunkwall, L., and Orho-Melander, M. (2017). The gut microbiome as a target for prevention and treatment of hyperglycaemia in type 2 diabetes: from current human evidence to future possibilities. Diabetologia 60, 943-951. doi: 10.1007/s00125-017-4278-3

Buse, J. B., DeFronzo, R. A., Rosenstock, J., Kim, T., Burns, C., Skare, S., et al. (2016). The primary glucose-lowering effect of metformin resides in the gut, not the circulation: results from short-term pharmacokinetic and 12-week dose-ranging studies. Diabetes Care 39, 198-205. doi: 10.2337/dc15-0488

Canfora, E. E., Jocken, J. W., and Blaak, E. E. (2015). Short-chain fatty acids in control of body weight and insulin sensitivity. Nat. Rev. Endocrinol. 11, 577-591. doi: 10.1038/nrendo.2015.128

Cani, P. D. (2018). Human gut microbiome: hopes, threats and promises. Gut 67, 1716-1725. doi: 10.1136/gutjnl-2018-316723

Caspary, W. F., and Creutzfeldt, W. (1975). Inhibition of bile salt absorption by blood-sugar lowering biguanides. Diabetologia 11, 113-117. doi: 10.1007/BF00429833

Chambers, E. S., Viardot, A., Psichas, A., Morrison, D. J., Murphy, K. G., ZacVarghese, S. E., et al. (2015). Effects of targeted delivery of propionate to the human colon on appetite regulation, body weight maintenance and adiposity in overweight adults. Gut 64, 1744-1754. doi: 10.1136/gutjnl-2014-307913

\section{ACKNOWLEDGMENTS}

We would like to thank Editage (www.editage.cn) for english language editing.

Chauveau, P., Fouque, D., Combe, C., and Aparicio, M. (2013). [Evolution of the diet from the paleolithic to today: progress or regress?]. Nephrol. Ther 9, 202-208. doi: 10.1016/j.nephro.2013.03.011

Chelakkot, C., Choi, Y., Kim, D. K., Park, H. T., Ghim, J., Kwon, Y., et al. (2018). Akkermansia muciniphila-derived extracellular vesicles influence gut permeability through the regulation of tight junctions. Exp. Mol. Med. 50:e450. doi: 10.1038/emm.2017.282

Chen, J. M., Chang, C. W., Lin, Y. C., Horng, J. T., and Sheu, W. H. (2014). Acarbose treatment and the risk of cardiovascular disease in type 2 diabetic patients: a nationwide seven-year follow-up study. J. Diabetes Res. 2014:812628. doi: 10.1155/2014/812628

Chiasson, J. L., Josse, R. G., Gomis, R., Hanefeld, M., Karasik, A., and Laakso, M. (2003). Acarbose treatment and the risk of cardiovascular disease and hypertension in patients with impaired glucose tolerance: the STOP-NIDDM trial. JAMA 290, 486-494. doi: 10.1001/jama.290.4.486

Clemente, J. C., Ursell, L. K., Parfrey, L. W., and Knight, R. (2012). The impact of the gut microbiota on human health: an integrative view. Cell 148, 1258-1270. doi: 10.1016/j.cell.2012.01.035

Costello, E. K., Stagaman, K., Dethlefsen, L., Bohannan, B. J., and Relman, D. A. (2012). The application of ecological theory toward an understanding of the human microbiome. Science 336, 1255-1262. doi: 10.1126/science.1224203

Croyal, M., Saulnier, P. J., Aguesse, A., Gand, E., Ragot, S., Roussel, R., et al. (2020). Plasma trimethylamine N-oxide and risk of cardiovascular events in patients with type 2 diabetes. J. Clin. Endocrinol. Metab. 105:dgaal88. doi: 10.1210/clinem/dgaa188

Curtiss, L. K., and Tobias, P. S. (2009). Emerging role of toll-like receptors in atherosclerosis. J. Lipid Res. 50 (Suppl.), S340-S345. doi: 10.1194/jlr.R800056-JLR200

Dao, M. C., Everard, A., Aron-Wisnewsky, J., Sokolovska, N., Prifti, E., Verger, E. O., et al. (2016). Akkermansia muciniphila and improved metabolic health during a dietary intervention in obesity: relationship with gut microbiome richness and ecology. Gut 65, 426-436. doi: 10.1136/gutjnl-2014-308778

de la Cuesta-Zuluaga, J., Mueller, N. T., Corrales-Agudelo, V., Velasquez-Mejia, E. P., Carmona, J. A., Abad, J. M., et al. (2017). Metformin is associated with higher relative abundance of mucin-degrading Akkermansia muciniphila and several short-chain fatty acid-producing microbiota in the gut. Diabetes Care 40, 54-62. doi: 10.2337/dc16-1324

de Vadder, F., Kovatcheva-Datchary, P., Zitoun, C., Duchampt, A., Bäckhed, F., and Mithieux, G. (2016). Microbiota-produced succinate improves glucose homeostasis via intestinal gluconeogenesis. Cell Metab. 24, 151-157. doi: 10.1016/j.cmet.2016.06.013

DECODE Study Group, the European Diabetes Epidemiology Group (2001). Glucose tolerance and cardiovascular mortality: comparison of fasting and 2-hour diagnostic criteria. Arch. Intern. Med. 161, 397-405. doi: 10.1001/archinte.161.3.397

DeFronzo, R. A., and Ferrannini, E. (1991). Insulin resistance. A multifaceted syndrome responsible for NIDDM, obesity, hypertension, dyslipidemia, and atherosclerotic cardiovascular disease. Diabetes Care 14, 173-194. doi: $10.2337 /$ diacare.14.3.173

den Besten, G., Bleeker, A., Gerding, A., van Eunen, K., Havinga, R., van Dijk, T. H., et al. (2015). Short-chain fatty acids protect against high-fat diet-induced obesity via a PPARgamma-dependent switch from lipogenesis to fat oxidation. Diabetes 64, 2398-2408. doi: 10.2337/db14-1213

Duca, F. A., Cote, C. D., Rasmussen, B. A., Zadeh-Tahmasebi, M., Rutter, G. A., Filippi, B. M., et al. (2015). Metformin activates a duodenal Ampk-dependent pathway to lower hepatic glucose production in rats. Nat. Med. 21, 506-511. doi: 10.1038/nm.3787

Eckburg, P. B., Bik, E. M., Bernstein, C. N., Purdom, E., Dethlefsen, L., Sargent, M., et al. (2005). Diversity of the human intestinal microbial flora. Science 308, 1635-1638. doi: 10.1126/science. 1110591 
Eliasson, B., Cederholm, J., Eeg-Olofsson, K., Svensson, A. M., Zethelius, B., and Gudbjornsdottir, S. (2011). Clinical usefulness of different lipid measures for prediction of coronary heart disease in type 2 diabetes: a report from the Swedish National diabetes register. Diabetes Care 34, 2095-2100. doi: $10.2337 / \mathrm{dc} 11-0209$

Emoto, T., Yamashita, T., Sasaki, N., Hirota, Y., Hayashi, T., So, A., et al. (2016). Analysis of gut microbiota in coronary artery disease patients: a possible link between gut microbiota and coronary artery disease. J. Atheroscler. Thromb. 23, 908-921. doi: 10.5551/jat.32672

Esposito, K., Nappo, F., Marfella, R., Giugliano, G., Giugliano, F., Ciotola, M., et al. (2002). Inflammatory cytokine concentrations are acutely increased by hyperglycemia in humans: role of oxidative stress. Circulation 106, 2067-2072. doi: 10.1161/01.CIR.0000034509.14906.AE

Estaki, M., Pither, J., Baumeister, P., Little, J. P., Gill, S. K., Ghosh, S., et al. (2016). Cardiorespiratory fitness as a predictor of intestinal microbial diversity and distinct metagenomic functions. Microbiome 4:42. doi: 10.1186/s40168-016-0189-7

Everard, A., Belzer, C., Geurts, L., Ouwerkerk, J. P., Druart, C., Bindels, L. B., et al. (2013). Cross-talk between Akkermansia muciniphila and intestinal epithelium controls diet-induced obesity. Proc. Natl. Acad. Sci. U.S.A. 110, 9066-9071. doi: $10.1073 /$ pnas. 1219451110

Foretz, M., Guigas, B., and Viollet, B. (2019). Understanding the glucoregulatory mechanisms of metformin in type 2 diabetes mellitus. Nat. Rev. Endocrinol. 15, 569-589. doi: 10.1038/s41574-019-0242-2

Forslund, K., Hildebrand, F., Nielsen, T., Falony, G., Le Chatelier, E., Sunagawa, S., et al. (2015). Disentangling type 2 diabetes and metformin treatment signatures in the human gut microbiota. Nature 528, 262-266. doi: 10.1038/nature15766

Fujisaka, S., Avila-Pacheco, J., Soto, M., Kostic, A., Dreyfuss, J. M., Pan, H., et al. (2018). Diet, genetics, and the gut microbiome drive dynamic changes in plasma metabolites. Cell Rep. 22, 3072-3086. doi: 10.1016/j.celrep.2018.02.060

Gao, Z., Yin, J., Zhang, J., Ward, R. E., Martin, R. J., Lefevre, M., et al. (2009). Butyrate improves insulin sensitivity and increases energy expenditure in mice. Diabetes 58, 1509-1517. doi: 10.2337/db08-1637

Geng, D. F., Jin, D. M., Wu, W., Fang, C., and Wang, J. F. (2011). Effect of alphaglucosidase inhibitors on the progression of carotid intima-media thickness: a meta-analysis of randomized controlled trials. Atherosclerosis 218, 214-219. doi: 10.1016/j.atherosclerosis.2011.05.004

Goncalves, P., Araujo, J. R., and Di Santo, J. P. (2018). A cross-talk between microbiota-derived short-chain fatty acids and the host mucosal immune system regulates intestinal homeostasis and inflammatory bowel disease. Inflamm. Bowel Dis. 24, 558-572. doi: 10.1093/ibd/izx029

Green, J. B., Bethel, M. A., Armstrong, P. W., Buse, J. B., Engel, S. S., Garg, J., et al. (2015). Effect of sitagliptin on cardiovascular outcomes in type 2 diabetes. $N$. Engl. J. Med. 373, 232-242. doi: 10.1056/NEJMoa1501352

Grice, E. A., and Segre, J. A. (2012). The human microbiome: our second genome. Annu. Rev. Genomics Hum. Genet 13, 151-170. doi: 10.1146/annurev-genom-090711-163814

Grundy, S. M., Cleeman, J. I., Merz, C. N., Brewer, H. B. Jr., Clark, L. T., Hunninghake, D. B., et al. (2004). Implications of recent clinical trials for the National cholesterol education program adult treatment panel III guidelines. Circulation 110, 227-239. doi: 10.1161/01.CIR.0000133317.49796.0E

Gu, Y., Wang, X., Li, J., Zhang, Y., Zhong, H., Liu, R., et al. (2017). Analyses of gut microbiota and plasma bile acids enable stratification of patients for antidiabetic treatment. Nat. Commun. 8, 1785-1785. doi: 10.1038/s41467-017-01682-2

Hai-xia, L. I. U., Jing, L. I., Ben, L. I. U., Dan-dan, L. I. U., Yong-juan, S., Ping, Z., et al. (2011). Effect of acarbose on fecal bifidobacteria content in patients with type 2 diabetes mellitus. Chin. J. Endocrinol. Metab. 27, 928-931. doi: 10.1007/s13300-017-0226-y

Han, Y., Xie, H., Liu, Y., Gao, P., Yang, X., and Shen, Z. (2019). Effect of metformin on all-cause and cardiovascular mortality in patients with coronary artery diseases: a systematic review and an updated meta-analysis. Cardiovasc. Diabetol. 18:96. doi: 10.1186/s12933-019-0900-7

Hanefeld, M. (2007). Cardiovascular benefits and safety profile of acarbose therapy in prediabetes and established type 2 diabetes. Cardiovasc. Diabetol. 6:20. doi: 10.1186/1475-2840-6-20

Hanefeld, M., Cagatay, M., Petrowitsch, T., Neuser, D., Petzinna, D., and Rupp, M. (2004). Acarbose reduces the risk for myocardial infarction in type 2 diabetic patients: meta-analysis of seven long-term studies. Eur. Heart J. 25, 10-16. doi: 10.1016/S0195-668X (03)00468-8

He, X., Zheng, N., He, J., Liu, C., Feng, J., Jia, W., et al. (2017). Gut microbiota modulation attenuated the hypolipidemic effect of simvastatin in high-fat/cholesterol-diet fed mice. J. Proteome Res. 16, 1900-1910. doi: 10.1021/acs.jproteome.6b00984

Hillman, E. T., Lu, H., Yao, T., and Nakatsu, C. H. (2017). Microbial ecology along the gastrointestinal tract. Microbes Environ. 32, 300-313. doi: 10.1264/jsme2.ME17017

Holman, R. R., Coleman, R. L., Chan, J. C. N., Chiasson, J. L., Feng, H., Ge, J., et al. (2017). Effects of acarbose on cardiovascular and diabetes outcomes in patients with coronary heart disease and impaired glucose tolerance (ACE): a randomised, double-blind, placebo-controlled trial. Lancet Diabetes Endocrinol. 5, 877-886. doi: 10.1016/S2213-8587 (17)30309-1

Holman, R. R., Paul, S. K., Bethel, M. A., Matthews, D. R., and Neil, H. A. (2008). 10-year follow-up of intensive glucose control in type 2 diabetes. N. Engl. J. Med. 359, 1577-1589. doi: 10.1056/NEJMoa0806470

Holst, J. J., and Deacon, C. F. (1998). Inhibition of the activity of dipeptidylpeptidase IV as a treatment for type 2 diabetes. Diabetes 47, 1663-1670. doi: 10.2337/diabetes.47.11.1663

Hong, J., Zhang, Y., Lai, S., Lv, A., Su, Q., Dong, Y., et al. (2013). Effects of metformin versus glipizide on cardiovascular outcomes in patients with type 2 diabetes and coronary artery disease. Diabetes Care 36, 1304-1311. doi: $10.2337 / \mathrm{dc} 12-0719$

Ishibashi, T. (2019). Therapeutic efficacy of molecular hydrogen: a new mechanistic insight. Curr. Pharm. Des. 25, 946-955. doi: 10.2174/1381612825666190506123038

Isomaa, B., Almgren, P., Tuomi, T., Forsen, B., Lahti, K., Nissen, M., et al. (2001). Cardiovascular morbidity and mortality associated with the metabolic syndrome. Diabetes Care 24, 683-689. doi: 10.2337/diacare.24.4.683

Jadhav, S., Ferrell, W., Greer, I. A., Petrie, J. R., Cobbe, S. M., and Sattar, N. (2006). Effects of metformin on microvascular function and exercise tolerance in women with angina and normal coronary arteries: a randomized, double-blind, placebo-controlled study. J. Am. Coll. Cardiol. 48, 956-963. doi: 10.1016/j.jacc.2006.04.088

Jefferis, B. J. M. H., Papacosta, O., Owen, C. G., Wannamethee, S. G., Humphries, S. E., Woodward, M., et al. (2011). Interleukin 18 and coronary heart disease: prospective study and systematic review. Atherosclerosis 217, 227-233. doi: 10.1016/j.atherosclerosis.2011.03.015

Jiao, N., Baker, S. S., Nugent, C. A., Tsompana, M., Cai, L., Wang, Y., et al. (2018). Gut microbiome may contribute to insulin resistance and systemic inflammation in obese rodents: a meta-analysis. Physiol. Genomics 50, 244-254. doi: 10.1152/physiolgenomics.00114.2017

Jie, Z., Xia, H., Zhong, S. L., Feng, Q., Li, S., Liang, S., et al. (2017). The gut microbiome in atherosclerotic cardiovascular disease. Nat. Commun. 8:845. doi: 10.1038/s41467-017-00900-1

Joyce, S. A., and Gahan, C. G. (2017). Disease-associated changes in bile acid profiles and links to altered gut microbiota. Dig. Dis. 35, 169-177. doi: 10.1159/000450907

Kaddurah-Daouk, R., Baillie, R. A., Zhu, H., Zeng, Z. B., Wiest, M. M., Nguyen, U. T., et al. (2011). Enteric microbiome metabolites correlate with response to simvastatin treatment. PLoS ONE 6:e25482. doi: 10.1371/journal.pone.0025482

Karbownik, M., Stasiak, M., Zygmunt, A., Zasada, K., and Lewiński, A. (2006). Protective effects of melatonin and indole-3-propionic acid against lipid peroxidation, caused by potassium bromate in the rat kidney. Cell Biochem. Funct. 24, 483-489. doi: 10.1002/cbf.1321

Karlson, B. W., Wiklund, O., Palmer, M. K., Nicholls, S. J., Lundman, P., and Barter, P. J. (2016). Variability of low-density lipoprotein cholesterol response with different doses of atorvastatin, rosuvastatin, and simvastatin: results from VOYAGER. Eur. Heart J. Cardiovasc. Pharmacother. 2, 212-217. doi: 10.1093/ehjcvp/pvw006

Karlsson, F. H., Tremaroli, V., Nookaew, I., Bergstrom, G., Behre, C. J., Fagerberg, B., et al. (2013). Gut metagenome in European women with normal, impaired and diabetic glucose control. Nature 498, 99-103. doi: 10.1038/nature12198

Kawano, H., Motoyama, T., Hirashima, O., Hirai, N., Miyao, Y., Sakamoto, T., et al. (1999). Hyperglycemia rapidly suppresses flow-mediated endotheliumdependent vasodilation of brachial artery. J. Am. Coll. Cardiol. 34, 146-154. doi: 10.1016/S0735-1097 (99)00168-0 
Khan, T. J., Ahmed, Y. M., Zamzami, M. A., Mohamed, S. A., Khan, I., Baothman, O. A. S., et al. (2018a). Effect of atorvastatin on the gut microbiota of high fat diet-induced hypercholesterolemic rats. Sci. Rep. 8:662. doi: 10.1038/s41598-017-19013-2

Khan, T. J., Ahmed, Y. M., Zamzami, M. A., Siddiqui, A. M., Khan, I., Baothman, O. A. S., et al. (2018b). Atorvastatin treatment modulates the gut microbiota of the hypercholesterolemic patients. OMICS 22, 154-163. doi: 10.1089/omi.2017.0130

Khat, D. Z., and Husain, M. (2018). Molecular mechanisms underlying the cardiovascular benefits of SGLT2i and GLP-1RA. Curr. Diab. Rep. 18:45. doi: 10.1007/s11892-018-1011-7

Kikuchi, K., Ben Othman, M., and Sakamoto, K. (2018). Sterilized bifidobacteria suppressed fat accumulation and blood glucose level. Biochem. Biophys. Res. Commun. 501, 1041-1047. doi: 10.1016/j.bbrc.2018.05.105

Kim, J., Lee, H., An, J., Song, Y., Lee, C. K., Kim, K., et al. (2019). Alterations in gut microbiota by statin therapy and possible intermediate effects on hyperglycemia and hyperlipidemia. Front. Microbiol. 10:1947. doi: 10.3389/fmicb.2019.01947

Kimura, I., Ozawa, K., Inoue, D., Imamura, T., Kimura, K., Maeda, T., et al. (2013). The gut microbiota suppresses insulin-mediated fat accumulation via the short-chain fatty acid receptor GPR43. Nat. Commun. 4:1829. doi: $10.1038 /$ ncomms 2852

Koeth, R. A., Levison, B. S., Culley, M. K., Buffa, J. A., Wang, Z., Gregory, J. C., et al. (2014). $\gamma$-Butyrobetaine is a proatherogenic intermediate in gut microbial metabolism of L-carnitine to TMAO. Cell Metab. 20, 799-812. doi: 10.1016/j.cmet.2014.10.006

Koh, A., de Vadder, F., Kovatcheva-Datchary, P., and Backhed, F. (2016). From dietary fiber to host physiology: short-chain fatty acids as key bacterial metabolites. Cell 165, 1332-1345. doi: 10.1016/j.cell.2016.05.041

Kooy, A., de Jager, J., Lehert, P., Bets, D., Wulffelé, M. G., Donker, A. J., et al. (2009). Long-term effects of metformin on metabolism and microvascular and macrovascular disease in patients with type 2 diabetes mellitus. Arch. Intern. Med. 169, 616-625. doi: 10.1001/archinternmed.2009.20

Kulkarni, S., Xavier, D., George, B., Umesh, S., Fathima, S., and Bantwal, G. (2018). Effect of intensive lifestyle modification \& metformin on cardiovascular risk in prediabetes: a pilot randomized control trial. Indian J. Med. Res. 148, 705-712. doi: 10.4103/ijmr.IJMR_1201_17

Lahnwong, S., Chattipakorn, S. C., and Chattipakorn, N. (2018). Potential mechanisms responsible for cardioprotective effects of sodiumglucose co-transporter 2 inhibitors. Cardiovasc. Diabetol. 17:101. doi: 10.1186/s12933-018-0745-5

Lam, V., Su, J., Hsu, A., Gross, G. J., Salzman, N. H., and Baker, J. E. (2016). Intestinal microbial metabolites are linked to severity of myocardial infarction in rats. PLoS ONE 11:e0160840. doi: 10.1371/journal.pone.0160840

Le Vee, M., Lecureur, V., Stieger, B., and Fardel, O. (2009). Regulation of drug transporter expression in human hepatocytes exposed to the proinflammatory cytokines tumor necrosis factor-alpha or interleukin-6. Drug Metab. Dispos. 37, 685-693. doi: 10.1124/dmd.108.023630

Lee, D. M., Battson, M. L., Jarrell, D. K., Hou, S., Ecton, K. E., Weir, T. L., et al. (2018). SGLT2 inhibition via dapagliflozin improves generalized vascular dysfunction and alters the gut microbiota in type 2 diabetic mice. Cardiovasc. Diabetol. 17:62. doi: 10.1186/s12933-018-0708-x

Lee, H., Kim, J., An, J., Lee, S., Choi, D., Kong, H., et al. (2019). Downregulation of IL-18 expression in the gut by metformin-induced gut microbiota modulation. Immune Netw. 19:e28. doi: 10.4110/in.2019.19.e28

Li, J., Lin, S., Vanhoutte, P. M., Woo, C. W., and Xu, A. (2016). Akkermansia muciniphila protects against atherosclerosis by preventing metabolic endotoxemia-induced inflammation in Apoe-/- mice. Circulation 133, 2434-2446. doi: 10.1161/CIRCULATIONAHA.115.019645

Liao, X., Song, L., Zeng, B., Liu, B., Qiu, Y., Qu, H., et al. (2019). Alteration of gut microbiota induced by DPP-4i treatment improves glucose homeostasis. EBioMedicine 44, 665-674. doi: 10.1016/j.ebiom.2019. 03.057

Lin, H. V., Frassetto, A., Kowalik, E. J. Jr., Nawrocki, A. R., Lu, M. M., Kosinski, J. R., et al. (2012). Butyrate and propionate protect against dietinduced obesity and regulate gut hormones via free fatty acid receptor 3independent mechanisms. PLOS ONE 7:e35240. doi: 10.1371/journal.pone.00 35240
Liu, Y., Song, X., Zhou, H., Zhou, X., Xia, Y., Dong, X., et al. (2018). Gut microbiome associates with lipid-lowering effect of rosuvastatin in vivo. Front. Microbiol. 9:530. doi: 10.3389/fmicb.2018.00530

Loh, M., Zhou, L., Ng, H. K., and Chambers, J. C. (2019). Epigenetic disturbances in obesity and diabetes: epidemiological and functional insights. Mol Metab. 27, S33-S41. doi: 10.1016/j.molmet.2019.06.011

Madamanchi, N. R., Hakim, Z. S., and Runge, M. S. (2005). Oxidative stress in atherogenesis and arterial thrombosis: the disconnect between cellular studies and clinical outcomes. J. Thromb. Haemost. 3, 254-267. doi: 10.1111/j.1538-7836.2004.01085.x

Madsen, M. S. A., Holm, J. B., Palleja, A., Wismann, P., Fabricius, K., Rigbolt, K. et al. (2019). Metabolic and gut microbiome changes following GLP-1 or dual GLP-1/GLP-2 receptor agonist treatment in diet-induced obese mice. Sci. Rep. 9:15582. doi: 10.1038/s41598-019-52103-X

Marso, S. P., Bain, S. C., Consoli, A., Eliaschewitz, F. G., Jodar, E., Leiter, L. A., et al. (2016a). Semaglutide and cardiovascular outcomes in patients with type 2 diabetes. N. Engl. J. Med. 375, 1834-1844. doi: 10.1056/NEJMoa1607141

Marso, S. P., Daniels, G. H., Brown-Frandsen, K., Kristensen, P., Mann, J. F., Nauck, M. A., et al. (2016b). Liraglutide and cardiovascular outcomes in type 2 diabetes. N. Engl. J. Med. 375, 311-322. doi: 10.1056/NEJMoa1603827

Matsubara, T., Li, F., and Gonzalez, F. J. (2013). FXR signaling in the enterohepatic system. Mol. Cell. Endocrinol. 368, 17-29. doi: 10.1016/j.mce.2012.05.004

Mazzone, T., Chait, A., and Plutzky, J. (2008). Cardiovascular disease risk in type 2 diabetes mellitus: insights from mechanistic studies. Lancet 371, 1800-1809. doi: 10.1016/S0140-6736 (08)60768-0

McAdam-Marx, C., Mukherjee, J., Bellows, B. K., Unni, S., Ye, X., Iloeje, U., et al. (2014). Evaluation of the relationship between weight change and glycemic control after initiation of antidiabetic therapy in patients with type 2 diabetes using electronic medical record data. Diabetes Res. Clin. Pract. 103, 402-411. doi: 10.1016/j.diabres.2013.12.038

Medzhitov, R. (2007). Recognition of microorganisms and activation of the immune response. Nature 449, 819-826. doi: 10.1038/nature06246

Meier, J. J. (2012). GLP-1 receptor agonists for individualized treatment of type 2 diabetes mellitus. Nat. Rev. Endocrinol. 8, 728-742. doi: $10.1038 /$ nrendo.2012.140

Mohan, M., Al-Talabany, S., McKinnie, A., Mordi, I. R., Singh, J. S. S., Gandy, S. J., et al. (2019). A randomized controlled trial of metformin on left ventricular hypertrophy in patients with coronary artery disease without diabetes: the MET-REMODEL trial. Eur. Heart J. 40, 3409-3417. doi: 10.1093/eurheartj/ehz203

Monami, M., Zannoni, S., Pala, L., Silverii, A., Andreozzi, F., Sesti, G., et al. (2017). Effects of glucagon-like peptide-1 receptor agonists on mortality and cardiovascular events: a comprehensive meta-analysis of randomized controlled trials. Int. J. Cardiol. 240, 414-421. doi: 10.1016/j.ijcard.2017.03.163

Montandon, S. A., and Jornayvaz, F. R. (2017). Effects of antidiabetic drugs on gut microbiota composition. Genes. 8:250. doi: 10.3390/genes 8100250

Mooradian, A. D. (2009). Dyslipidemia in type 2 diabetes mellitus. Nat. Clin. Pract. Endocrinol. Metab. 5, 150-159. doi: 10.1038/ncpendmet1066

Morgan, C. L., Mukherjee, J., Jenkins-Jones, S., Holden, S. E., and Currie, C. J. (2014). Association between first-line monotherapy with sulphonylurea versus metformin and risk of all-cause mortality and cardiovascular events: a retrospective, observational study. Diabetes Obes. Metab. 16, 957-962. doi: 10.1111/dom.12302

Mulvihill, E. E., and Drucker, D. J. (2014). Pharmacology, physiology, and mechanisms of action of dipeptidyl peptidase-4 inhibitors. Endocr. Rev. 35, 992-1019. doi: 10.1210/er.2014-1035

Napolitano, A., Miller, S., Nicholls, A. W., Baker, D., Van Horn, S., Thomas, E., et al. (2014). Novel gut-based pharmacology of metformin in patients with type 2 diabetes mellitus. PLoS ONE 9:e100778. doi: 10.1371/journal.pone.0100778

Neal, B., Perkovic, V., Mahaffey, K. W., de Zeeuw, D., Fulcher, G., Erondu, N., et al. (2017). Canagliflozin and cardiovascular and renal events in type 2 diabetes. $N$. Engl. J. Med. 377, 644-657. doi: 10.1056/NEJMoa1611925

Nemet, I., Saha, P. P., Gupta, N., Zhu, W., Romano, K. A., Skye, S. M., et al. (2020). A cardiovascular disease-linked gut microbial metabolite acts via adrenergic receptors. Cell 180, 862-877.e822. doi: 10.1016/j.cell.2020.02.016

Neves, A. L., Coelho, J., Couto, L., Leite-Moreira, A., and Roncon-Albuquerque, R. Jr. (2013). Metabolic endotoxemia: a molecular link between obesity and cardiovascular risk. J. Mol. Endocrinol. 51, R51-R64. doi: 10.1530/JME-13-0079 
Nicholson, J. K., Holmes, E., Kinross, J., Burcelin, R., Gibson, G., Jia, W., et al. (2012). Host-gut microbiota metabolic interactions. Science 336, 1262-1267. doi: $10.1126 /$ science. 1223813

Ning, F., Tuomilehto, J., Pyorala, K., Onat, A., Soderberg, S., and Qiao, Q. (2010). Cardiovascular disease mortality in Europeans in relation to fasting and 2h plasma glucose levels within a normoglycemic range. Diabetes Care 33, 2211-2216. doi: $10.2337 / \mathrm{dc} 09-2328$

Node, K., and Inoue, T. (2009). Postprandial hyperglycemia as an etiological factor in vascular failure. Cardiovasc. Diabetol. 8:23. doi: 10.1186/1475-2840-8-23

Olivares, M., Schüppel, V., Hassan, A. M., Beaumont, M., Neyrinck, A. M., Bindels, L. B., et al. (2018). The potential role of the dipeptidyl peptidase-4-like activity from the gut microbiota on the host health. Front. Microbiol. 9:1900. doi: $10.3389 /$ fmicb. 2018.01900

Pahwa, R., Balderas, M., Jialal, I., Chen, X., Luna, R. A., and Devaraj, S. (2017). Gut microbiome and inflammation: a study of diabetic inflammasome-knockout mice. J. Diabetes Res. 2017:6519785. doi: 10.1155/2017/6519785

Palm, N. W., de Zoete, M. R., and Flavell, R. A. (2015). Immunemicrobiota interactions in health and disease. Clin. Immunol. 159, 122-127. doi: 10.1016/j.clim.2015.05.014

Pernicova, I., and Korbonits, M. (2014). Metformin-mode of action and clinical implications for diabetes and cancer. Nat. Rev. Endocrinol. 10, 143-156. doi: 10.1038 /nrendo.2013.256

Philpott, D. J., Sorbara, M. T., Robertson, S. J., Croitoru, K., and Girardin, S. E. (2014). NOD proteins: regulators of inflammation in health and disease. Nat. Rev. Immunol. 14, 9-23. doi: 10.1038/nri3565

Plovier, H., Everard, A., Druart, C., Depommier, C., van Hul, M., Geurts, L., et al. (2017). A purified membrane protein from Akkermansia muciniphila or the pasteurized bacterium improves metabolism in obese and diabetic mice. Nat. Med. 23, 107-113. doi: 10.1038/nm.4236

Pols, T. W., Nomura, M., Harach, T., Lo Sasso, G., Oosterveer, M. H., Thomas, C., et al. (2011). TGR5 activation inhibits atherosclerosis by reducing macrophage inflammation and lipid loading. Cell Metab. 14, 747-757. doi: 10.1016/j.cmet.2011.11.006

Preiss, D., Lloyd, S. M., Ford, I., McMurray, J. J., Holman, R. R., Welsh, P., et al. (2014). Metformin for non-diabetic patients with coronary heart disease (the CAMERA study): a randomised controlled trial. Lancet Diabetes Endocrinol. 2, 116-124. doi: 10.1016/S2213-8587 (13)70152-9

Qin, J., Li, R., Raes, J., Arumugam, M., Burgdorf, K. S., Manichanh, C., et al. (2010). A human gut microbial gene catalogue established by metagenomic sequencing. Nature 464, 59-65. doi: 10.1038/nature08821

Qin, J., Li, Y., Cai, Z., Li, S., Zhu, J., Zhang, F., et al. (2012). A metagenomewide association study of gut microbiota in type 2 diabetes. Nature 490, 55-60. doi: $10.1038 /$ nature 11450

Rena, G., and Lang, C. C. (2018). Repurposing metformin for cardiovascular disease. Circulation 137, 422-424. doi: 10.1161/CIRCULATIONAHA.117.031735

Ripatti, S., Tikkanen, E., Orho-Melander, M., Havulinna, A. S., Silander, K., Sharma, A., et al. (2010). A multilocus genetic risk score for coronary heart disease: case-control and prospective cohort analyses. Lancet 376, 1393-1400. doi: 10.1016/S0140-6736 (10)61267-6

Roager, H. M., and Licht, T. R. (2018). Microbial tryptophan catabolites in health and disease. Nat. Commun. 9:3294 doi: 10.1038/s41467-018-05470-4

Samuel, B. S., Shaito, A., Motoike, T., Rey, F. E., Backhed, F., Manchester, J. K., et al. (2008). Effects of the gut microbiota on host adiposity are modulated by the short-chain fatty-acid binding G protein-coupled receptor, Gpr41. Proc. Natl. Acad. Sci. U.S.A. 105, 16767-16772. doi: 10.1073/pnas.0808567105

Scheen, A. J. (2018). Cardiovascular effects of new oral glucose-lowering agents: DPP-4 and SGLT-2 inhibitors. Circ. Res. 122, 1439-1459. doi: 10.1161/CIRCRESAHA.117.311588

Schramm, C. (2018). Bile acids, the microbiome, immunity, and liver tumors. $N$. Engl. J. Med. 379, 888-890. doi: 10.1056/NEJMcibr1807106

Scirica, B. M., Bhatt, D. L., Braunwald, E., Steg, P. G., Davidson, J., Hirshberg, B., et al. (2013). Saxagliptin and cardiovascular outcomes in patients with type 2 diabetes mellitus. N. Engl. J. Med. 369, 1317-1326. doi: 10.1056/NEJMoa1307684

Shin, N. R., Lee, J. C., Lee, H. Y., Kim, M. S., Whon, T. W., Lee, M. S., et al. (2014). An increase in the Akkermansia spp. population induced by metformin treatment improves glucose homeostasis in diet-induced obese mice. Gut 63, 727-735. doi: 10.1136/gutjnl-2012-303839

Sonnenburg, J. L., and Backhed, F. (2016). Diet-microbiota interactions as moderators of human metabolism. Nature 535, 56-64. doi: $10.1038 /$ nature 18846

Stancu, C., and Sima, A. (2001). Statins: mechanism of action and effects. J. Cell. Mol. Med. 5, 378-387. doi: 10.1111/j.1582-4934.2001.tb00172.x

Standl, E., Theodorakis, M. J., Erbach, M., Schnell, O., and Tuomilehto, J. (2014). On the potential of acarbose to reduce cardiovascular disease. Cardiovasc. Diabetol. 13:81. doi: 10.1186/1475-2840-13-81

Staudinger, J. L., Goodwin, B., Jones, S. A., Hawkins-Brown, D., MacKenzie, K. I., LaTour, A., et al. (2001). The nuclear receptor PXR is a lithocholic acid sensor that protects against liver toxicity. Proc. Natl. Acad. Sci. U.S.A. 98, 3369-3374. doi: 10.1073/pnas.051551698

Stepensky, D., Friedman, M., Raz, I., and Hoffman, A. (2002). Pharmacokineticpharmacodynamic analysis of the glucose-lowering effect of metformin in diabetic rats reveals first-pass pharmacodynamic effect. Drug Metab. Dispos. 30, 861-868. doi: 10.1124/dmd.30.8.861

Stone, N. J., Robinson, J. G., Lichtenstein, A. H., Bairey Merz, C. N., Blum, C. B., Eckel, R. H., et al. (2014). 2013 ACC/AHA guideline on the treatment of blood cholesterol to reduce atherosclerotic cardiovascular risk in adults: a report of the American college of cardiology/American heart association task force on practice guidelines. J. Am. Coll. Cardiol. 63, 2889-2934. doi: 10.1016/j.jacc.2013.11.002

Su, B., Liu, H., Li, J., Sunli, Y., Liu, B., Liu, D., et al. (2015). Acarbose treatment affects the serum levels of inflammatory cytokines and the gut content of bifidobacteria in Chinese patients with type 2 diabetes mellitus. J. Diabetes 7, 729-739. doi: 10.1111/1753-0407.12232

Sui, Y., Xu, J., Rios-Pilier, J., and Zhou, C. (2011). Deficiency of PXR decreases atherosclerosis in apoE-deficient mice. J. Lipid Res. 52, 1652-1659. doi: 10.1194/jlr.M017376

Sun, L., Xie, C., Wang, G., Wu, Y., Wu, Q., Wang, X., et al. (2018). Gut microbiota and intestinal FXR mediate the clinical benefits of metformin. Nat. Med. 24, 1919-1929. doi: 10.1038/s41591-018-0222-4

Suzuki, Y., Sano, M., Hayashida, K., Ohsawa, I., Ohta, S., and Fukuda, K. (2009). Are the effects of alpha-glucosidase inhibitors on cardiovascular events related to elevated levels of hydrogen gas in the gastrointestinal tract? FEBS Lett. 583, 2157-2159. doi: 10.1016/j.febslet.2009.05.052

Tang, W. H., Kitai, T., and Hazen, S. L. (2017). Gut microbiota in cardiovascular health and disease. Circ. Res. 120, 1183-1196. doi: 10.1161/CIRCRESAHA.117.309715

Tang, W. H., Wang, Z., Levison, B. S., Koeth, R. A., Britt, E. B., Fu, X., et al. (2013). Intestinal microbial metabolism of phosphatidylcholine and cardiovascular risk. N. Engl. J. Med. 368, 1575-1584. doi: 10.1056/NEJMoa1109400

Tang, W. H. W., Backhed, F., Landmesser, U., and Hazen, S. L. (2019). Intestinal microbiota in cardiovascular health and disease: JACC state-of-the-art review. J. Am. Coll. Cardiol. 73, 2089-2105. doi: 10.1016/j.jacc.2019.03.024

Topping, D. L., and Clifton, P. M. (2001). Short-chain fatty acids and human colonic function: roles of resistant starch and nonstarch polysaccharides. Physiol. Rev. 81, 1031-1064. doi: 10.1152/physrev.2001.81.3.1031

Touyz, R. M. (2004). Reactive oxygen species, vascular oxidative stress, and redox signaling in hypertension: what is the clinical significance? Hypertension 44, 248-252. doi: 10.1161/01.HYP.0000138070.47616.9d

UKPDS Group, the UK Prospective Diabetes Study Group (1998). Effect of intensive blood-glucose control with metformin on complications in overweight patients with type 2 diabetes (UKPDS 34). UK Prospective Diabetes Study (UKPDS) Group. Lancet 352, 854-865. doi: 10.1016/S0140-6736(98)07037-8

van Stee, M. F., de Graaf, A. A., and Groen, A. K. (2018). Actions of metformin and statins on lipid and glucose metabolism and possible benefit of combination therapy. Cardiovasc. Diabetol. 17:94. doi: 10.1186/s12933-018-0738-4

Veiga, P., Juste, C., Lepercq, P., Saunier, K., Beguet, F., and Gerard, P. (2005). Correlation between faecal microbial community structure and cholesterol-tocoprostanol conversion in the human gut. FEMS Microbiol. Lett. 242, 81-86. doi: 10.1016/j.femsle.2004.10.042

Vilsboll, T., Christensen, M., Junker, A. E., Knop, F. K., and Gluud, L. L. (2012). Effects of glucagon-like peptide-1 receptor agonists on weight loss: systematic 
review and meta-analyses of randomised controlled trials. BMJ 344:d7771. doi: 10.1136/bmj.d7771

Viollet, B., Guigas, B., Sanz Garcia, N., Leclerc, J., Foretz, M., and Andreelli, F. (2012). Cellular and molecular mechanisms of metformin: an overview. Clin. Sci. 122, 253-270. doi: 10.1042/CS20110386

Wahlstrom, A., Sayin, S. I., Marschall, H. U., and Backhed, F. (2016). Intestinal crosstalk between bile acids and microbiota and its impact on host metabolism. Cell Metab. 24, 41-50. doi: 10.1016/j.cmet.2016. 05.005

Wang, L., Li, P., Tang, Z., Yan, X., and Feng, B. (2016). Structural modulation of the gut microbiota and the relationship with body weight: compared evaluation of liraglutide and saxagliptin treatment. Sci. Rep. 6:33251. doi: 10.1038/ srep33251

Wang, L., Wang, Y., Wang, H., Zhou, X., Wei, X., Xie, Z., et al. (2018a). The influence of the intestinal microflora to the efficacy of Rosuvastatin. Lipids Health Dis. 17:151. doi: 10.1186/s12944-018-0801-x

Wang, Z., Klipfell, E., Bennett, B. J., Koeth, R., Levison, B. S., Dugar, B., et al. (2011). Gut flora metabolism of phosphatidylcholine promotes cardiovascular disease. Nature 472, 57-63. doi: 10.1038/nature09922

Wang, Z., Roberts, A. B., Buffa, J. A., Levison, B. S., Zhu, W., Org, E., et al. (2015). Non-lethal inhibition of gut microbial trimethylamine production for the treatment of atherosclerosis. Cell 163, 1585-1595. doi: $10.1016 /$ j.cell.2015.11.055

Wang, Z., Saha, S., van Horn, S., Thomas, E., Traini, C., Sathe, G., et al. (2018b). Gut microbiome differences between metformin- and liraglutide-treated T2DM subjects. Endocrinol. Diabetes Metab. 1:e00009. doi: 10.1002/edm2.9

Wen, L., and Duffy, A. (2017). Factors influencing the gut microbiota, inflammation, and type 2 diabetes. J. Nutr. 147, 1468s-1475s. doi: 10.3945/jn.116.240754

Weng, J., Soegondo, S., Schnell, O., Sheu, W. H., Grzeszczak, W., Watada, H., et al. (2015). Efficacy of acarbose in different geographical regions of the world: analysis of a real-life database. Diabetes Metab. Res. Rev. 31, 155-167. doi: $10.1002 / \mathrm{dmrr} .2576$

Whalen, K., Miller, S., and Onge, E. S. (2015). The role of sodium-glucose cotransporter 2 inhibitors in the treatment of type 2 diabetes. Clin. Ther. 37, 1150-1166. doi: 10.1016/j.clinthera.2015.03.004

White, W. B., Cannon, C. P., Heller, S. R., Nissen, S. E., Bergenstal, R. M., Bakris, G. L., et al. (2013). Alogliptin after acute coronary syndrome in patients with type 2 diabetes. N. Engl. J. Med. 369, 1327-1335. doi: 10.1056/NEJMoa1305889

Whiting, D. R., Guariguata, L., Weil, C., and Shaw, J. (2011). IDF diabetes atlas: global estimates of the prevalence of diabetes for 2011 and 2030. Diabetes Res. Clin. Pract. 94, 311-321. doi: 10.1016/j.diabres.2011.10.029

Winer, D. A., Luck, H., Tsai, S., and Winer, S. (2016). The intestinal immune system in obesity and insulin resistance. Cell Metab. 23, 413-426. doi: 10.1016/j.cmet.2016.01.003

Wiviott, S. D., Raz, I., Bonaca, M. P., Mosenzon, O., Kato, E. T., Cahn, A., et al. (2019). Dapagliflozin and cardiovascular outcomes in type 2 diabetes. N. Engl. J. Med. 380, 347-357. doi: 10.1056/NEJMoa1812389
Wlodarska, M., Luo, C., Kolde, R., d’Hennezel, E., Annand, J. W., Heim, C. E., et al. (2017). Indoleacrylic acid produced by commensal peptostreptococcus species suppresses inflammation. Cell Host Microbe 22, 25-37.e26. doi: 10.1016/j.chom.2017.06.007

Wolf, G. (2006). Gut microbiota: a factor in energy regulation. Nutr. Rev. 64, 47-50. doi: 10.1111/j.1753-4887.2006.tb00173.x

Wu, H., Esteve, E., Tremaroli, V., Khan, M. T., Caesar, R., Manneras-Holm, L., et al. (2017). Metformin alters the gut microbiome of individuals with treatmentnaive type 2 diabetes, contributing to the therapeutic effects of the drug. Nat. Med. 23, 850-858. doi: 10.1038/nm.4345

Yan, X., Feng, B., Li, P., Tang, Z., and Wang, L. (2016). Microflora disturbance during progression of glucose intolerance and effect of sitagliptin: an animal study. J. Diabetes Res 2016:2093171. doi: 10.1155/2016/2093171

Zhang, Q., Xiao, X., Li, M., Yu, M., Ping, F., Zheng, J., et al. (2017). Vildagliptin increases butyrate-producing bacteria in the gut of diabetic rats. PLoS ONE 12:e0184735. doi: 10.1371/journal.pone.0184735

Zhang, Q., Xiao, X., Zheng, J., Li, M., Yu, M., Ping, F., et al. (2018). Featured article: structure moderation of gut microbiota in liraglutide-treated diabetic male rats. Exp. Biol. Med. 243, 34-44. doi: 10.1177/1535370217743765

Zhang, Y., Wang, X., Vales, C., Lee, F. Y., Lee, H., Lusis, A. J., et al. (2006). FXR deficiency causes reduced atherosclerosis in Ldlr-/- mice. Arterioscler. Thromb. Vasc. Biol. 26, 2316-2321. doi: 10.1161/01.ATV.0000235697.35431.05

Zhao, L., Chen, Y., Xia, F., Abudukerimu, B., Zhang, W., Guo, Y., et al. (2018). A glucagon-like peptide-1 receptor agonist lowers weight by modulating the structure of gut microbiota. Front. Endocrinol. 9:233. doi: $10.3389 /$ fendo.2018.00233

Zhu, W., Gregory, J. C., Org, E., Buffa, J. A., Gupta, N., Wang, Z., et al. (2016). Gut microbial metabolite TMAO enhances platelet hyperreactivity and thrombosis risk. Cell 165, 111-124. doi: 10.1016/j.cell.2016.02.011

Zilov, A. V., Abdelaziz, S. I., AlShammary, A., Al Zahrani, A., Amir, A., Assaad Khalil, S. H., et al. (2019). Mechanisms of action of metformin with special reference to cardiovascular protection. Diabetes Metab. Res. Rev. 35:e3173. doi: $10.1002 / \mathrm{dmrr} .3173$

Zinman, B., Lachin, J. M., and Inzucchi, S. E. (2016). Empagliflozin, cardiovascular outcomes, and mortality in type 2 diabetes. N. Engl. J. Med. 374:1094. doi: 10.1056/NEJMc1600827

Conflict of Interest: The authors declare that the research was conducted in the absence of any commercial or financial relationships that could be construed as a potential conflict of interest.

Copyright (c) 2020 Ding, Tian, Li, Lian, Zhao, Wei, Han, Zheng, Gao, Yang, Fang and Tong. This is an open-access article distributed under the terms of the Creative Commons Attribution License (CC BY). The use, distribution or reproduction in other forums is permitted, provided the original author(s) and the copyright owner(s) are credited and that the original publication in this journal is cited, in accordance with accepted academic practice. No use, distribution or reproduction is permitted which does not comply with these terms. 